\title{
The origin and evolution of dense regions in the ISM, and their role in spectral features
}

\author{
Diego Falceta-Gonçalves
}

\author{
Universidade de São Paulo, Rua Arlindo Bettio 1000, CEP 03828-000, São Paulo, Brazil \\ email: dfalceta@usp.br
}

\begin{abstract}
In this work we discuss the turbulent evolution of molecular clouds and the formation of dense structures within. Typically, the clumps evolution occurs apart from the secular evolution of the turbulent mother cloud due to its high density and large inertia. Despite of current theoretical assumptions we show, by means of numerical simulations, that the clump lifetimes are greater than previously thought by more than an order of magnitude. The presence of dense and long-lived clumps modifies the spectral line profiles of clouds, which are strongly related to the determination of Larson's relations. We address the main modifications of these if a realistic distribution of dense structures is taken into account.
\end{abstract}

Keywords. ISM: kinematics and dynamics — magnetohydrodynamics (MHD)

Numerical simulations of the ISM turbulence reveal that gravitationally unbound clumps may be long lived $\left(\tau>10^{7} \mathrm{yrs}\right)$, despite of the generally assumed timescales of dense structures (see Fig1a) (Falceta-Gonçalves \& Lazarian 2011). The reason is their large densities, compared to the surrounding medium. The increased life timescale of dense clumps has severe implications in massive star formation theories, for example.

Another interesting implication is related to the dynamics of the ISM. Dense clumps dynamics may become independent from the ISM large scale turbulence. However, most of the emission lines used to estimate flow velocities in molecular clouds, as well as the Larson's relations, which are used to predict turbulent properties, have their fluxes dominated by the dense regions. We showed that using line profiles observed from dense regions to study the turbulent properties of the ISM is not straightforward (FalcetaGonçalves et al. 2010).

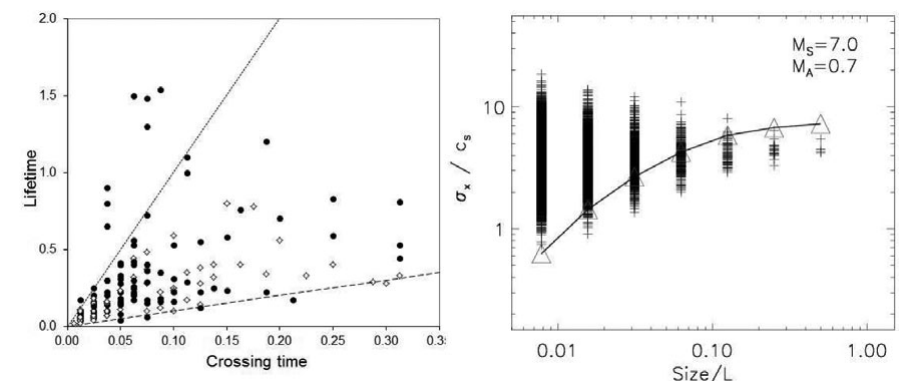

Figure 1. Left: life timescale of dense clumps (Falceta-Gonçalves \& Lazarian 2011). Right: relation of length scale to velocity dispersion of line profiles (Falceta-Gonçalves et al. 2010).

\section{References}

Falceta-Gonçalves D., Lazarian A. \& Houde M. 2010, ApJ, 713, 1376

Falceta-Gonçalves D.\& Lazarian A. 2011, ApJ, 735, 99 